\title{
Structure of the Mediator head module
}

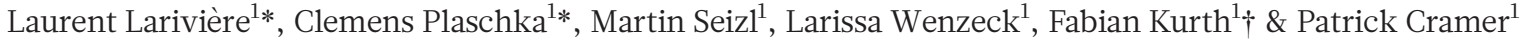

Gene transcription by RNA polymerase (Pol) II requires the coactivator complex Mediator. Mediator connects transcriptional regulators and Pol II, and is linked to human disease ${ }^{1-4}$. Mediator from the yeast Saccharomyces cerevisiae has a molecular mass of 1.4 megadaltons and comprises 25 subunits that form the head, middle, tail and kinase modules ${ }^{5-7}$. The head module constitutes one-half of the essential Mediator core ${ }^{8}$, and comprises the conserved ${ }^{9}$ subunits Med6, Med8, Med11, Med17, Med18, Med20 and Med22. Recent X-ray analysis of the $S$. cerevisiae head module at $4.3 \AA$ resolution led to a partial architectural model with three submodules called neck, fixed jaw and moveable jaw ${ }^{10}$. Here we determine de novo the crystal structure of the head module from the fission yeast Schizosaccharomyces pombe at $3.4 \AA$ resolution. Structure solution was enabled by new structures of Med6 and the fixed jaw, and previous structures of the moveable jaw ${ }^{11}$ and part of the neck ${ }^{12}$, and required deletion of Med20. The $S$. pombe head module resembles the head of a crocodile with eight distinct elements, of which at least four are mobile. The fixed jaw comprises tooth and nose domains, whereas the neck submodule contains a helical spine and one limb, with shoulder, arm and finger elements. The arm and the essential shoulder contact other parts of Mediator. The jaws and a central joint are implicated in interactions with Pol II and its carboxy-terminal domain, and the joint is required for transcription in vitro. The $S$. pombe head module structure leads to a revised model of the $S$. cerevisiae module, reveals a high conservation and flexibility, explains known mutations, and provides the basis for unravelling a central mechanism of gene regulation.

Extending our structural analysis of Mediator ${ }^{13}$, we determined the crystal structure of a bacterially expressed Med6 variant (S. pombe (Sp) residues 9-180) at $2.7 \AA$ resolution (Supplementary Figs 1, 2a and Supplementary Table 1). The structure revealed a core domain with a five-stranded antiparallel $\beta$-sheet, two pairs of $\alpha$-helices flanking a conserved groove, and a flexible C-terminal helix $\alpha 5$ (Fig. 1a, b and Supplementary Fig. 3). Bacterial expression also provided the head subcomplex Med17C-Med11C-Med22C, which consists of C-terminal regions in Med17, Med11 and Med22 (ref. 12) and constitutes the fixed jaw $^{10}$ (see Methods and Supplementary Fig. 2b). The crystal structure at 3.0 A resolution (Supplementary Table 1) revealed two subdomains we call 'tooth' and 'nose' (Fig. 1c). The tooth contains Med11C, Med22C and Med17C residues 383-541 and 594-611. It forms a five-stranded $\beta$-sheet that is flanked by five helices, three from Med17C and one each from Med11C and Med22C. The nose comprises Med17C residues 542-580 and 612-687 and forms another five-stranded $\beta$-sheet with two flanking helices.

We next prepared the entire $S$. cerevisiae $(S c)$ head module by coexpressing its seven subunits in Escherichia coli (see Methods and Supplementary Fig. 2c). The purified module (Fig. 2a) supported activator- and promoter-dependent transcription from three different promoters in vitro (Fig. $2 \mathrm{~b}$ and Supplementary Note 1). Crystals of the $S c$ head module were obtained in 2007 , but persistently diffracted to low resolution. We therefore prepared the head module from Sp (Supplementary Fig. 2d). The purified module (Fig. 2a) did not crystallize, but crystals were obtained for a variant lacking three non-essential parts, Med6 residues 181-216, Med17 residues 1-76 and Med20 (Fig. 2a and Supplementary Fig. 2e).

We determined the $S p$ head module structure de novo by multiple isomorphous replacement with anomalous scattering (see Methods and Supplementary Fig. 4a). Modelling required selenomethionine sequence markers (Supplementary Fig. 4b) and the four available structures of subcomplexes (Supplementary Fig. 5a-e). Diffraction data to $3.4 \AA$ resolution (Supplementary Table 2) resulted in excellent electron density (Supplementary Fig. 4c, d) and a refined structure that has a free $R$ factor $\left(R_{\text {free }}\right)$ of $25.8 \%$ (Supplementary Table 2$)$ and contains $87 \%$ of the residues in the variant.

The $S p$ head module structure reveals an asymmetric multiprotein assembly that resembles the head of a crocodile with one limb (Figs $2 \mathrm{c}$ and 3a). The structure reveals all folds and previously unobserved regions, and is partitioned into eight elements (Fig. 3a, b). The neck submodule is formed by Med6, Med8 and parts of Med11, Med17 and Med22, and consists of 'spine', 'shoulder', 'arm' and 'finger' elements (Fig. 3a, b and Supplementary Figs 6-8). The spine comprises seven helices, six forming a long bundle (Med8 $\alpha 5$, Med17 $\alpha 3$, Med11NMed22N (ref. 12)), and a seventh perpendicular helix (Med8 $\alpha 4)$. The arm consists of a short four-helix bundle (Med6 $\alpha 5$, Med8 $\alpha 1 / 2$, Med17 $\alpha 1$ ), two pairs of helices (Med17 $\eta 1 / \alpha 2$, Med $8 \alpha 2^{\prime} / \alpha 3$ ), and a two-stranded sheet (Med8 $\beta 1$, Med17 $\beta 1)$. The arm binds the shoulder, which consists of the Med6 core (Fig. 1a). The finger is an exposed C-terminal $\beta$-hairpin in Med6 (Fig. 3a, d). The fixed and moveable
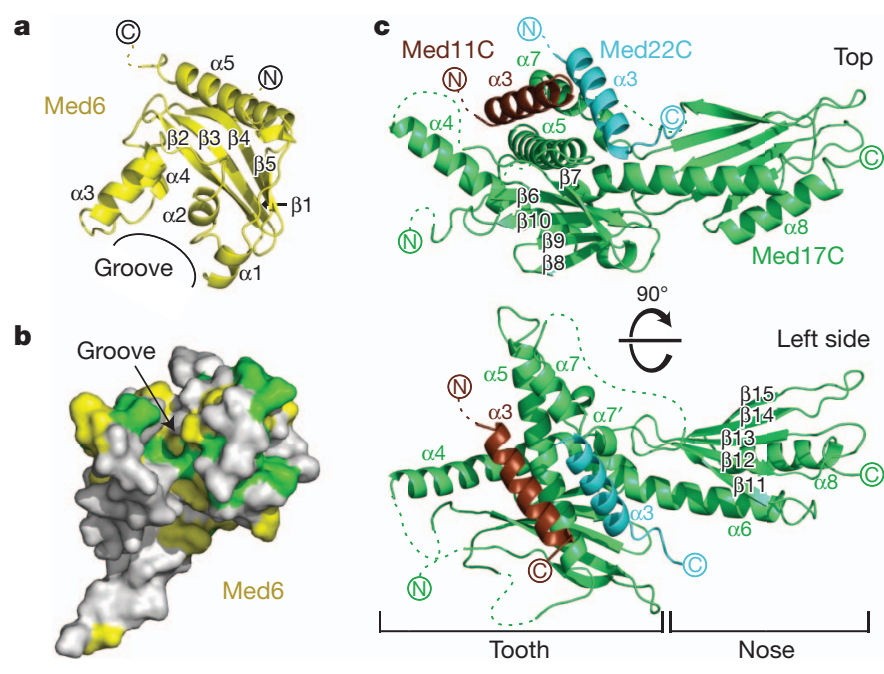

Figure $1 \mid$ Structures of Med6 and Med17C-Med11C-Med22C. a, Ribbon model of the Sp Med6 crystal structure. b, Conserved surface groove in Med6. Residues that are invariant or conserved among seven yeast species are in green or yellow, respectively (see Supplementary Fig. 1). c, Ribbon model of the Sc subcomplex Med17C-Med11C-Med22C crystal structure. 'Tooth' and 'nose' domains are indicated. Dashed lines indicate disordered regions. 
a

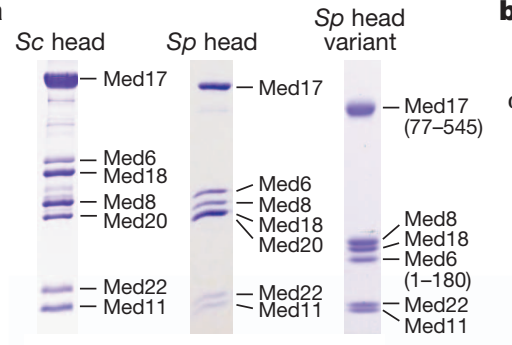

b

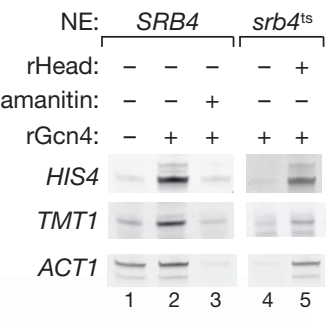

c

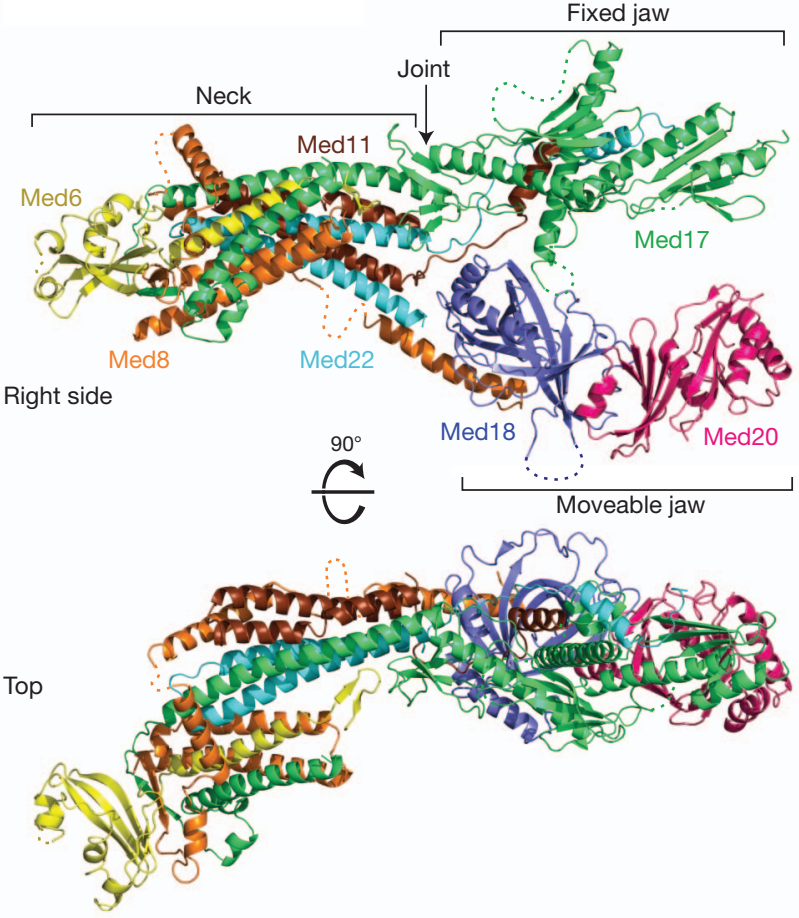

Figure $2 \mid$ Structure of $S p$ Mediator head module. a, SDS-polyacrylamide gel electrophoresis (PAGE) analysis of purified recombinant head modules of $S c$ (left), full-length $S p$ (middle) and the crystallized $S p$ variant (right). b, Recombinant $S c$ head module supports in vitro transcription from three different promoter types. Activity of nuclear extracts from SRB4 and srb4-138 $\left(\operatorname{srb} 4^{\mathrm{ts}}\right)$ strains was assessed on promoters HIS4, TMT1 and ACT1. Pol II-dependency was confirmed by $\alpha$-amanitin sensitivity $(0.04 \mathrm{mM}$, lane 3$)$. Addition of 5 pmol recombinant $S c$ head module (lane 5) to inactive $s r b 4^{\text {ts }}$ extracts (lane 4) restored activity. c, Two views of a ribbon representation of Sp Mediator head module structure. Subunits are in different colours. Subunit Med20 is not present in the crystals but was unambiguously modelled and positioned by superimposing the previous Sc Med18-Med20 heterodimer structure ${ }^{11}$.

jaws consist of subcomplexes Med17C-Med11C-Med22C (Figs 1c and 3a) and Med8C-Med18-Med20 (ref. 11), respectively.

Both jaws are connected to the spine. The fixed jaw is connected via the 'joint' that contains a four-stranded $\beta$-sheet formed by Med17 residues 215-259, and short linkers in Med11 and Med22. The joint may change its structure as it contains many conserved glycine and serine residues. Deletion of the Med17 part of the joint did not cause lethality in yeast, but essentially abolished activated transcription in vitro (Supplementary Fig. 9a, b). The moveable jaw binds the spine via Med8C, a helix extending from the Med18-Med20 heterodimer ${ }^{14}$ (Fig. 3a, d), and also contacts the joint and tooth. Conservation of the module surface is highest at the shoulder and arm (Fig. $3 \mathrm{c}$ and Supplementary Fig. 6), probably because this region binds the middle module ${ }^{15,16}$. Deletion of the shoulder caused lethality (Supplementary Fig. 9c).

We prepared a homology model for the Sc head module based on our structures of the $S p$ module and the $S c$ subcomplexes
Med17C-Med11C-Med22C, Med11N-Med22N (ref. 12) and Med8CMed18-Med20 (ref. 11). After minor adjustments, the model explained the published electron density and selenium positions ${ }^{10}$ (Supplementary Fig. 10a). Compared with the published model ${ }^{10}$, four $\alpha$-helices were swapped, a $\beta$-sheet was added in the tooth, and the amino acid register was adjusted for $46 \%$ of the residues outside the moveable jaw (Supplementary Fig. 10b). Thus the structures of the $S p$ and $S c$ head modules are well conserved although the structured regions show only $15 \%$ sequence identity (Supplementary Fig. 1). Sc-specific differences are found in six helices; three are shorter $(\operatorname{Med} 8 \alpha \mathrm{C}$, Med17 $\alpha 5, \operatorname{Med} 22$ $\alpha 1$ ), one is longer (Med11 $\alpha 1$ ) and two are rotated (Med17 $\alpha 7, \operatorname{Med} 22$ a3) (Supplementary Fig. 5b, d, e). Because of poor electron density ${ }^{10}$, the revised $S c$ model lacks the joint, part of the arm, the sheet in the shoulder, the finger and five protein linkers.

Structural comparisons reveal flexibility within the head module. The position of the shoulder changes in different $S p$ module crystals (Supplementary Fig. 11a), owing to a flexible connection to Med6 helix $\alpha 5$ (Supplementary Figs 3 and $5 \mathrm{c}$ ). The finger is mobile in the free Med6 structure. Flexibility of the moveable jaw is indicated by high B-factors (Supplementary Fig. 11b and Supplementary Note 2) and electron microscopic results ${ }^{17}$. Movement of the nose with respect to the tooth is revealed by comparison of the $S c$ model with the free fixed jaw structure (Supplementary Fig. 11c). Comparison of the Sp structure with the $S c$ model indicates that the neck can rotate with respect to the jaws (Supplementary Fig. 11e). The rotation may be accommodated by the joint, which uses conserved hydrophobic residues to anchor the tooth to the spine. Thus the head module contains at least four mobile peripheral elements, the shoulder, finger, moveable jaw and nose.

To explain the phenotypes of known mutations, we mapped 47 sites of $55 S c$ head module mutations onto the $S p$ structure (Fig. 4, Supplementary Fig. 1 and Supplementary Table 3). Most temperaturesensitive mutations map to protein cores or domain interfaces and apparently influence module stability (Fig. 4a). These include mutant srb4-138 that contains six Med17 mutations required for temperature sensitivity ${ }^{18}$, including mutations Ser226Pro and Phe649Ser that apparently destabilize the arm and nose, respectively. The human MED17 mutation Leu371Pro (residues 504 and 389 in Sc and Sp, respectively) is associated with infantile cerebral atrophy ${ }^{3}$ and perturbs MED17 helix $\alpha 5$ in the tooth. The temperature-sensitive phenotype for Med11 mutations Glu17Lys/Leu24Lys (ref. 12), Leu66Pro (refs 12, 19), and Glu92Ser (refs 12,19) is also explained by fold destabilizations. The mutation med6-ts 1 and mutations in the med6-ts 6 allele ${ }^{20}$ may destabilize the shoulder. Mutant med6-ts 2 contains six point mutations ${ }^{20}$, of which Gln49Leu changes the conserved surface implicated in binding other Mediator parts, Phe125Tyr changes the shoulder-arm interface, and Phe194Leu may destabilize the finger-spine interface.

The structure also explains how mutant phenotypes are suppressed by secondary mutations. The SRB4-101 mutation Glu286Lys rescues the med6-ts 2 phenotype ${ }^{20}$, apparently by creating a new salt bridge between Med17 helix $\alpha 3$ and Med6 helix $\alpha 5$. The MED6-101 mutation $^{21}$ may suppress the srb4-138 phenotype because mutation Asp152Tyr between the shoulder and arm compensates for decreased stability of the neck. The SRB6-201 (ref. 21) mutation Asn59His may also suppress the srb4-138 phenotype by stabilizing the neck.

Other genetic data implicate the fixed jaw in interactions with Pol II (Fig. 4b, Supplementary Fig. 1 and Supplementary Table 3). The head module interacts with Pol II near subunit Rpb3, and this contact is required for regulated transcription ${ }^{22,23}$. Mutations causing co-lethality with the Rpb3 mutation rpb3-2 map to the fixed jaw (Fig. 4b). Mutants med17-208 and med17-257 both contain the mutation Glu669Asp that is located on the nose surface and may abolish the head-polymerase interaction that is weakened by Rpb3 mutation. Mutant med17-sup1 rescues the phenotype of Rpb3 mutation Ala159Gly (ref. 22) and contains two mutations in the fixed jaw (Fig. 4b). Consistent with an interaction between the head module and the Pol II region around 


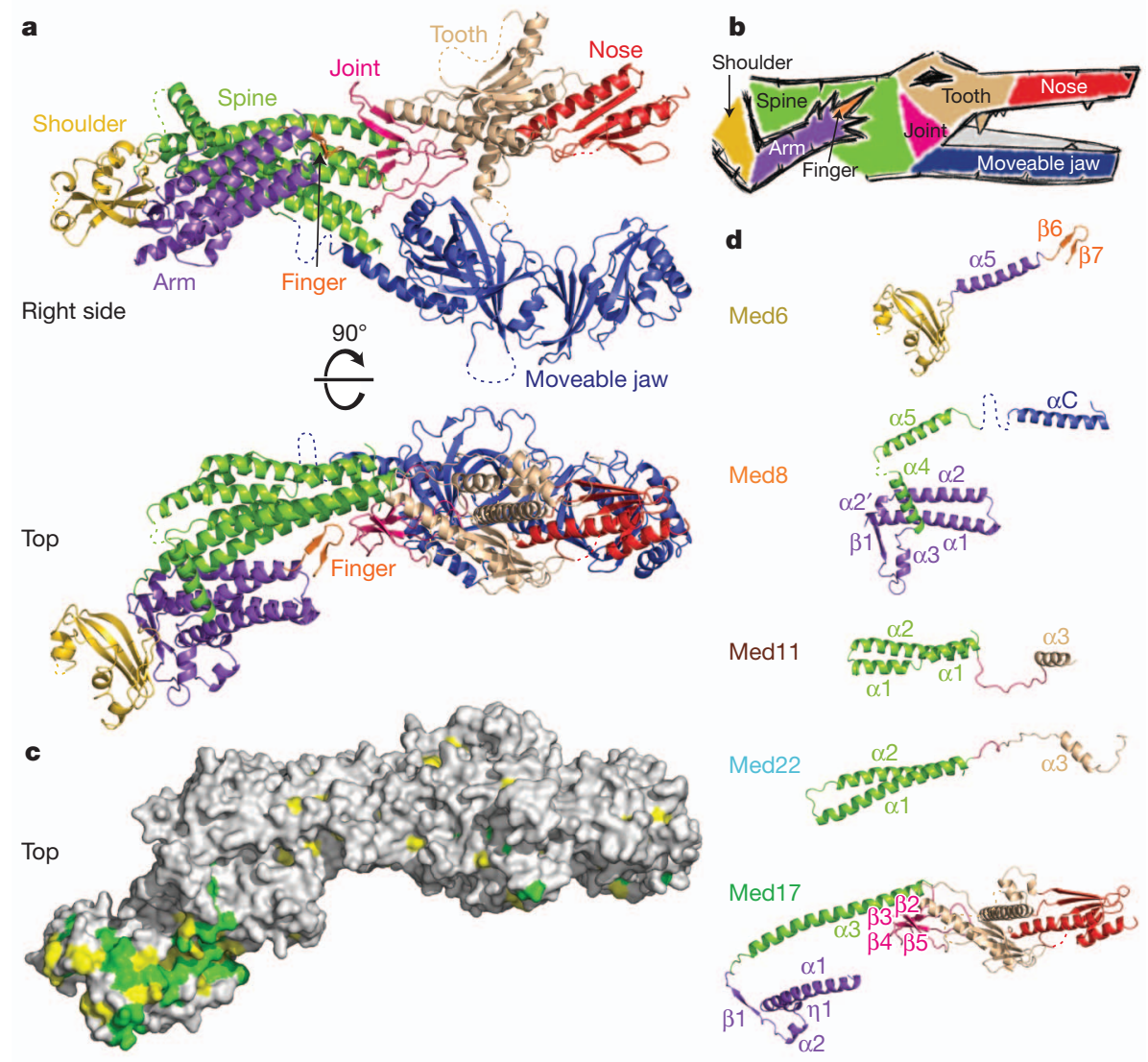

Figure 3 Structural elements and surface conservation. a, Eight structural elements in the Mediator head module. Ribbon model of the Sp head module structure is coloured accordingly (see also Supplementary Figs 6-8). b, The structure resembles the head of a crocodile with one limb. The eight structural elements received corresponding names, and previously introduced names for the three submodules neck, fixed jaw and moveable jaw ${ }^{10}$ are preserved.

a

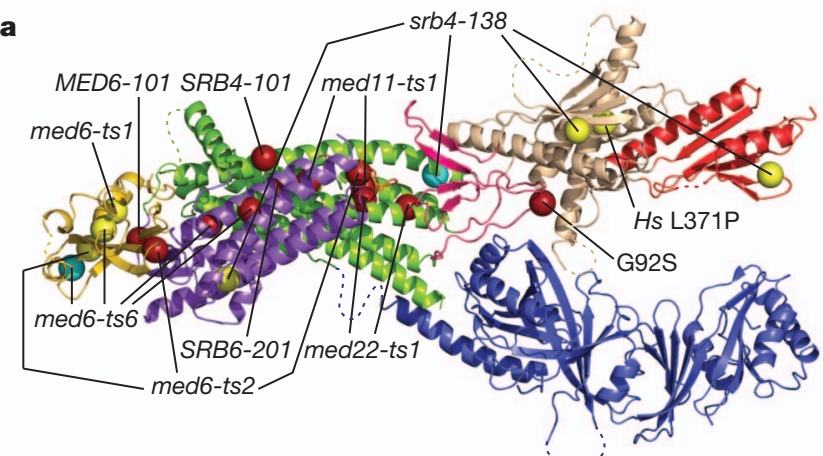

b

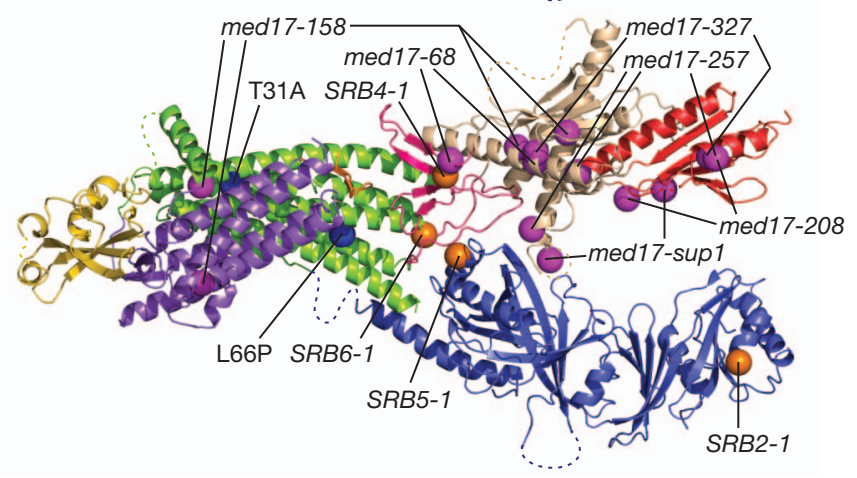

c) Surface conservation of the $S p$ Mediator head module. Residues that are invariant or conserved among seven yeast species (Supplementary Fig. 1) are in green or yellow, respectively (see also Supplementary Fig. 6). d, Ribbon representation of the five non-globular head subunits (top view) coloured according to structural elements as in a (see also Supplementary Figs 7 and 8).

Rpb3, a mutation in the adjacent polymerase dock domain has a coldsensitive phenotype that is rescued by $S R B$ mutations ${ }^{24}$ in the joint and moveable jaw (Supplementary Fig. 12, rpb1-14). Mutants med17-68, med17-158 and med17-327 all contain mutations that are predicted to destabilize the fixed jaw.

Mediator also interacts with the carboxy-terminal domain (CTD $)^{4,25}$ that extends from the polymerase core near the dock domain (Supplementary Fig. 12). CTD truncation causes a cold-sensitive phenotype that is rescued by mutations ${ }^{24,26,27}$ that map mainly to the joint (Fig. 4b). The mutation SRB4-1 maps to the joint-tooth interface, whereas the mutation SRB6-1 may stabilize the spine and adjacent joint. The mutation SRB5-1 in Med18 helix $\alpha 1$ may strengthen joint interaction with the moveable jaw, whereas mutation SRB2-1 alters the moveable jaw surface. These results suggest that the joint and moveable

Figure $4 \mid$ Head module integrity and interactions. a, Location of sites of $S c$ mutations that may influence module stability. Mutations were mapped onto the $S p$ head module structure using alignments shown in Supplementary Fig. 1. $\mathrm{C} \alpha$ atoms of mutated residues are shown as spheres. Mutations that may affect intrasubunit stability, intersubunit stability or with uncertain affects are in yellow, dark red or cyan, respectively. $\mathbf{b}$, Location of sites of $S c$ mutations that may influence interactions with the Pol II core, CTD and TFIIH. Mutations were mapped on the $S p$ head module using alignments shown in Supplementary Fig. 1, with $\mathrm{C} \alpha$ atoms represented as spheres. Mutations that suppress CTD truncation phenotypes, interact genetically with Rpb3 mutations or lead to a loss of interaction with TFIIH are shown as orange, magenta or blue spheres, respectively. A Med11 mutation in the spine, Thr31Ala, leads to reduced interaction with TFIIH (ref. 19). 
jaw are involved in CTD binding and/or that they are required for structural changes in Mediator that enable CTD binding. However, other Mediator regions probably contribute to interactions with the CTD because the head module is insufficient for CTD binding in $v$ itro $^{28}$. The flexibility and extended shape of the head module, which is $170 \AA$ long, $95 \AA$ high and $65 \AA$ wide, may allow for several interactions not only with Pol II but also with transcription factors TFIIB ${ }^{6,29}$, TFIIH $^{12,19}$ and the TATA box-binding protein ${ }^{11}$.

\section{METHODS SUMMARY}

To enable heterologous co-expression of head module subunits and their variant in E. coli, more than 300 expression vectors were tested. Expression vectors used in this work are shown in Supplementary Fig. 2. All proteins were expressed in E. coli BL21(DE3)RIL cells and purified by nickel affinity, anion exchange and sizeexclusion chromatography. Crystals were grown by vapour diffusion at $20^{\circ} \mathrm{C}$. $S p$ Med6 crystals were grown using reservoir solutions containing $100 \mathrm{mM}$ HEPES, $\mathrm{pH} 7.5$, and $400 \mathrm{mM}$ sodium citrate or $16 \%(\mathrm{v} / \mathrm{v})$ tacsimate for native or selenomethionine-labelled protein, respectively. Sc Med17C-Med11C-Med22C crystals were grown in $100 \mathrm{mM}$ MES, pH 6.0, and $4 \mathrm{M}$ ammonium acetate. Sp head module crystals were grown in $50 \mathrm{mM} \mathrm{MES,} \mathrm{pH} \mathrm{6.0,} \mathrm{and} 1 \mathrm{M}$ ammonium sulphate. Diffraction data were collected under cryo-conditions at Swiss Light Source (SLS) beamlines X06SA and X06DA. The Med6 and Med17C-Med11C-Med22C structures were solved using selenomethionine labelling and single-wavelength anomalous dispersion phasing. The head module structure was solved by MIRAS using tantalum and ytterbium derivatives and 23 anomalous difference Fourier peaks in a selenomethionine-labelled crystal as sequence markers. Yeast nuclear extract preparation and transcription assays were performed as described ${ }^{12,30}$.

Full Methods and any associated references are available in the online version of the paper.

\section{Received 10 July; accepted 12 October 2012}

Published online 31 October 2012.

1. Conaway, R. C. \& Conaway, J. W. Origins and activity of the Mediator complex. Semin. Cell Dev. Biol. 22, 729-734 (2011).

2. Chen, W. \& Roeder, R. G. Mediator-dependent nuclear receptor function. Semin. Cell Dev. Biol. (2011).

3. Kaufmann, R. et al. Infantile cerebral and cerebellar atrophy is associated with a mutation in the MED17 subunit of the transcription preinitiation mediator complex. Am. J. Hum. Genet. 87, 667-670 (2010).

4. Myers, L. C. et al. The Med proteins of yeast and their function through the RNA polymerase II carboxy-terminal domain. Genes Dev. 12, 45-54 (1998).

5. Asturias, F. J., Jiang, Y. W., Myers, L. C., Gustafsson, C. M. \& Kornberg, R. D. Conserved structures of mediator and RNA polymerase II holoenzyme. Science 283, 985-987 (1999).

6. Kang, J. S. et al. The structural and functional organization of the yeast mediator complex. J. Biol. Chem. 276, 42003-42010 (2001).

7. Davis, J. A., Takagi, Y., Kornberg, R. \& Asturias, F. Structure of the yeast RNA polymerase II holoenzyme: mediator conformation and polymerase interaction. Mol. Cell 10, 409-415 (2002).

8. Liu, Y., Ranish, J. A., Aebersold, R. \& Hahn, S. Yeast nuclear extract contains two major forms of RNA polymerase II mediator complexes. J. Biol. Chem. 276, 7169-7175 (2001)

9. Bourbon, H. M. Comparative genomics supports a deep evolutionary origin for the large, four-module transcriptional mediator complex. Nucleic Acids Res. 36 3993-4008 (2008)

10. Imasaki, T. et al. Architecture of the Mediator head module. Nature 475, 240-243 (2011)

11. Larivière, L. et al. Structure and TBP binding of the Mediator head subcomplex Med8-Med18-Med20. Nature Struct. Mol. Biol. 13, 895-901 (2006)

12. Seizl, M., Lariviere, L., Pfaffeneder, T., Wenzeck, L. \& Cramer, P. Mediator head subcomplex Med11/22 contains a common helix bundle building block with a specific function in transcription initiation complex stabilization. Nucleic Acids Res. 39, 6291-6304 (2011).

13. Larivière, L., Seizl, M. \& Cramer, P. A structural perspective on Mediator function. Curr. Opin. Cell Biol. (2012).

14. Larivière, L. et al. Structure-system correlation identifies a gene regulatory Mediator submodule. Genes Dev. 22, 872-877 (2008).
15. Baumli, S, Hoeppner, S. \& Cramer, P. A conserved mediator hinge revealed in the structure of the MED7·MED21 (Med7·Srb7) heterodimer. J. Biol. Chem. 280, 18171-18178 (2005).

16. Guglielmi, B. et al. A high resolution protein interaction map of the yeast Mediator complex. Nucleic Acids Res. 32, 5379-5391 (2004).

17. Cai, G. et al. Mediator head module structure and functional interactions. Nature Struct. Mol. Biol. 17, 273-279 (2010).

18. Takagi, Y. \& Kornberg, R. D. Mediator as a general transcription factor.J. Biol. Chem 281, 80-89 (2006)

19. Esnault, C. etal. Mediator-dependent recruitment of TFIIH modules in preinitiation complex. Mol. Cell 31, 337-346 (2008).

20. Lee, Y. C. \& Kim, Y. J. Requirement for a functional interaction between mediator components Med6 and Srb4 in RNA polymerase II transcription. Mol. Cell. Biol. 18, 5364-5370 (1998)

21. Lee, T. I. et al. Interplay of positive and negative regulators in transcription initiation by RNA polymerase II holoenzyme. Mol. Cell. Biol. 18, 4455-4462 (1998).

22. Soutourina, J., Wydau, S., Ambroise, Y., Boschiero, C. \& Werner, M. Direct interaction of RNA polymerase II and mediator required for transcription in vivo. Science 331, 1451-1454 (2011).

23. Tan, Q., Linask, K. L., Ebright, R. H. \& Woychik, N. A. Activation mutants in yeast RNA polymerase II subunit RPB3 provide evidence for a structurally conserved surface required for activation in eukaryotes and bacteria. Genes Dev. 14, 339-348 (2000).

24. Thompson, C. M., Koleske, A. J., Chao, D. M. \& Young, R. A. A multisubunit complex associated with the RNA polymerase II CTD and TATA-binding protein in yeast. Cell 73, 1361-1375 (1993).

25. Näär, A. M., Taatjes, D. J., Zhai, W., Nogales, E. \& Tjian, R. Human CRSP interacts with RNA polymerase II CTD and adopts a specific CTD-bound conformation. Genes Dev. 16, 1339-1344 (2002)

26. Nonet, M. L. \& Young, R. A. Intragenic and extragenic suppressors of mutations in the heptapeptide repeat domain of Saccharomyces cerevisiae RNA polymerase II. Genetics 123, 715-724 (1989).

27. Koleske, A. J., Buratowski, S., Nonet, M. \& Young, R. A. A novel transcription factor reveals a functional link between the RNA polymerase II CTD and TFIID. Cell 69, 883-894 (1992)

28. Takagi, Y. et al. Head module control of mediator interactions. Mol. Cell 23, 355-364 (2006)

29. Baek, H. J., Kang, Y. K. \& Roeder, R. G. Human Mediator enhances basal transcription by facilitating recruitment of transcription factor IIB during preinitiation complex assembly. J. Biol. Chem. 281, 15172-15181 (2006).

30. Ranish, J. A., Yudkovsky, N. \& Hahn, S. Intermediates in formation and activity of the RNA polymerase II preinitiation complex: holoenzyme recruitment and a postrecruitment role for the TATA box and TFIIB. Genes Dev. 13, 49-63 (1999).

Supplementary Information is available in the online version of the paper.

Acknowledgements We thank S. Baumli, A. C. Cheung, A. Imhof and C. Schmidt for help. We acknowledge the crystallization facility at the Max Planck Institute of Biochemistry, Martinsried. Diffraction data were collected at the Swiss Light Source, Villigen, Switzerland. M.S. was supported by a Boehringer Ingelheim fellowship and the Elite Network of Bavaria. P.C. was supported by the Deutsche Forschungsgemeinschaft, SFB646, TR5, GraKo1721, SFB960, CIPSM, NIM, an Advanced Grant of the European Research Council, the LMUinnovativ project Bioimaging Network, the Vallee Foundation, and the Jung-Stiftung.

Author Contributions L.L. designed crystallization constructs, established expression and purification strategies for head modules, solved the Med6 and Med17C-Med11CMed22C structures, carried out crystallographic data analysis for Sp head module structure determination, built the Sc head module model, generated yeast strains, and performed in vivo studies. C.P. optimized Sp head module purification, purified and crystallized the $S p$ head module variants, and prepared heavy metal derivatives. L.L. and C.P. collected diffraction data for the head module and carried out model building and structural analysis. M.S. contributed to establishing expression and purification strategies and carried out transcription assays. L.W. provided technical help. F.K. optimized transcription assays. P.C. initiated and supervised the project. P.C., L.L., C.P. and M.S. prepared the manuscript.

Author Information Atomic coordinate files and structure factors were deposited in the Protein Data Bank under accession codes 4H61 (Sp Med6), 4H62 (Sc Med17CMed11C-Med22C) and 4H63 (Sp head module). See Supplementary Data for the coordinate file for the revised Sc head module model. Reprints and permissions information is available at www.nature.com/reprints. The authors declare no competing financial interests. Readers are welcome to comment on the online version of the paper. Correspondence and requests for materials should be addressed to L.L. (larivier@genzentrum.Imu.de) or P.C. (cramer@genzentrum.Imu.de). 


\section{METHODS}

Vectors and sequences. To enable heterologous co-expression of head module subunits and their variants in E. coli, more than 300 expression vectors were tested. Vectors used in this work are shown in Supplementary Fig. 2. Vectors containing a single T7 promoter are based on pET21, pET24 or pET28 vectors (Novagen). Vectors containing two T7 promoters are based on Duet vectors (Novagen). The head co-expression vector that contains three T7 promoters was constructed by concatenating two Duet vectors. Open reading frames (ORFs) were cloned sequentially into vectors. For polycistronic constructs, ribosomal-binding sites were introduced as described ${ }^{15}$. Sequences of all constructs are available on request. All proteins were expressed in E. coli BL21(DE3)RIL (Stratagene). Cells were grown in $\mathrm{LB}$ medium at $37^{\circ} \mathrm{C}$ to an attenuance $(D)$ of 0.5 at $600 \mathrm{~nm}$. Expression was induced with $0.5 \mathrm{mM}$ isopropyl- $\beta$-D-thiogalactoside (IPTG) for $16 \mathrm{~h}$ at $18{ }^{\circ} \mathrm{C}$. Sequence alignments were performed with MUSCLE ${ }^{31}$ and manually adjusted based on the presented structures. Graphical representation in Supplementary Fig. 1 was generated with ESPript ${ }^{32}$.

Sp Med6 crystal structure determination. For expression of Sp Med6 (residues 9-180), cells were transformed with the vector depicted in Supplementary Fig. 2a and the protein was purified as described ${ }^{12}$ except that a HiLoad 16/600 Superdex $200 \mathrm{pg}$ (GE Healthcare) was used for size exclusion chromatography. Crystals were grown at $20^{\circ} \mathrm{C}$ in hanging drops over reservoirs containing $100 \mathrm{mM}$ HEPES $\mathrm{pH} 7.5$, and $400 \mathrm{mM}$ sodium citrate. For crystallization of selenomethioninelabelled ${ }^{33}$ protein, sodium citrate was replaced by $16 \%(\mathrm{v} / \mathrm{v})$ tacsimate. Crystals were collected, cryo-protected by gradually adding glycerol to a final concentration of $30 \%(\mathrm{v} / \mathrm{v})$, and flash-frozen in liquid nitrogen. Native and selenomethione single-wavelength anomalous dispersion (SAD) diffraction data were collected at $100 \mathrm{~K}$ on a PILATUS 6M detector at the X06SA beamline at Swiss Light Source (SLS), Villigen, Switzerland. Data were processed with XDS and XSCALE ${ }^{34}$ Phenix.autosol ${ }^{35}$ was used to detect selenium sites, phase the structure, perform density modification, and for initial model building. The model was manually adjusted using COOT $^{36}$ and refined with Phenix.refine ${ }^{35}$, including high-resolution data ${ }^{37}$ because the resulting final model had a better $R_{\text {free }}$ value and stereochemistry. The refined structure has an $R_{\text {free }}$ value of $21.7 \%$ and shows very good stereochemistry (Supplementary Table 1). Ninety-six per cent of the residues fall in favoured regions of the Ramachandran plot and none of them is in disallowed regions ${ }^{38}$.

Sc Med17C-Med11C-Med22C crystal structure determination. Sc Med17CMed11C-Med22C was expressed in cells co-transformed with the two vectors depicted in Supplementary Fig. 2b and was purified as Sp Med6 above. For the Med11C-Med22C fusion construct, a linker with sequence GAGSGAGSG was inserted between the $\mathrm{C}$ terminus of Med11 and residue 96 of Med22. This covalent linker was essential for complex stability. Crystals were grown at $20^{\circ} \mathrm{C}$ in hanging drops over reservoirs containing $100 \mathrm{mM}$ MES, $\mathrm{pH} 6.0$, and $4 \mathrm{M}$ ammonium acetate. Crystals were collected, transferred to a solution containing $100 \mathrm{mM}$ MES, pH 6.0 and 5 M ammonium acetate, and flash-frozen in liquid nitrogen. Selenomethionine labelling and diffraction data collection were as above for $S p$ Med6. Data were processed with XDS and XSCALE ${ }^{34}$. SHELXC/D/E $\mathrm{E}^{39}$ was used to detect selenium sites, phase the structure, and perform density modification. The resulting electron density map allowed for building of most of the model with COOT $^{36}$. The model was refined with Phenix.refine ${ }^{35}$, including high-resolution data ${ }^{37}$ because the resulting final model had a better $R_{\text {free }}$ value and stereochemistry. The refined structure has a $R_{\text {free }}$ value of $23.3 \%$ and shows very good stereochemistry (Supplementary Table 1). Ninety-six of the residues fall in favoured regions of the Ramachandran plot and none of them is in disallowed regions ${ }^{38}$.

Preparation of recombinant $S c$ head module. $S c$ head module was expressed in cells transformed with the single vector depicted in Supplementary Fig. 2c. Cells were lysed by sonication in buffer A $(50 \mathrm{mM}$ HEPES-potassium hydroxide, $\mathrm{pH} 7.5,400 \mathrm{mM}$ potassium chloride, $10 \%$ (v/v) glycerol, $5 \mathrm{mM}$ dithiothreitol (DTT) ) containing protease inhibitors ${ }^{12}$. After centrifugation, the supernatant was loaded onto a $1 \mathrm{ml}$ HisTrap column (GE Healthcare) equilibrated in buffer B (50 mM HEPES-potassium hydroxide, $\mathrm{pH} 7.5,500 \mathrm{mM}$ potassium acetate, $10 \%$ (v/v) glycerol, $50 \mathrm{mM}$ imidazole, $5 \mathrm{mM}$ DTT). The complex was eluted with a linear gradient from $50 \mathrm{mM}$ to $300 \mathrm{mM}$ imidazole in buffer B. The Sc head module was further purified by anion exchange chromatography with a $1 \mathrm{ml} \mathrm{HiTrap} \mathrm{Q} \mathrm{HP}$ column (GE Healthcare). The column was equilibrated in buffer C (50 mM HEPES-potassium hydroxide, $\mathrm{pH} 7.5,150 \mathrm{mM}$ potassium acetate, 10\% (v/v) glycerol, 2 mM DTT), and proteins were eluted with a linear gradient from $150 \mathrm{mM}$ to $1.25 \mathrm{M}$ potassium acetate in buffer $\mathrm{C}$. Fractions containing head module were applied to a HiLoad 16/600 Superdex 200 pg (GE healthcare) size exclusion column equilibrated in buffer D (20 mM HEPES-potassium hydroxide, pH 7.5, $150 \mathrm{mM}$ potassium acetate, $10 \%$ (v/v) glycerol, $2 \mathrm{mM}$ DTT). The $S c$ head module was concentrated to $5 \mathrm{mg} \mathrm{ml}^{-1}$, flash-frozen, and stored at $-80^{\circ} \mathrm{C}$. The identity of the polypeptides was confirmed by mass spectrometry. Static light scattering showed that the complex contains one copy of each subunit (not shown). The
$S c$ head module variant lacking the Med17 part of the joint $\left(\operatorname{Med} 17_{\Delta 321-369}\right)$ was expressed and purified the same way.

Yeast strains and functional assays. Plasmids pRS316-SRB4 and pRS316-MED6 were generated by cloning the respective ORF plus 500 base pairs (bp) upstream and $300 \mathrm{bp}$ downstream sequence into pRS316 (ATCC; URA3 marker). Plasmids pRS315-SRB4, pRS315-srb4 ${ }^{\text {ts }}$, pRS315-med17 $4321-369$, pRS315-MED6, pRS315med6 ${ }_{1-225}$ and pRS315-med6 $6_{163-295}$ were generated by cloning the respective wild-type or mutant ORF plus 500 bp upstream and 300 bp downstream sequence into pRS315 (ATCC; LEU2 marker). The heterozygous MED17/med17A and $M E D 6 / m e d 6 \Delta S c$ yeast strains were obtained from Euroscarf and transformed with pRS316-SRB4 and pRS316-MED6, respectively. Diploids were sporulated, tetrads were dissected and analysed, and a shuffle strain was selected. To assess functionality of mutants, pRS315 constructs were transformed into the respective shuffle strain. Equal amounts of freshly grown yeast cells in SC (-Ura - Leu) medium were resuspended in water and tenfold dilutions were spotted on 5 - fluoroorotic acid (5-FOA) and SC (-Ura - Leu) plates. Isogenic SRB4 and $s r b 4^{\text {ts }}$ strains used for nuclear extract preparation were obtained by transforming the MED17 shuffle strain with plasmids pRS315-SRB4 or pRS315-srb $4^{\text {ts }}$ and streaking transformants twice on 5-FOA plates. Nuclear extracts were prepared from 31 of yeast cultures as described ${ }^{12,30}$. In vitro transcription and analysis by primer extension were performed as described ${ }^{12}$. Primer extension was done using the same 5'-Cy5-labelled oligonucleotide (5' -TTCACCAGTGAGACGGGCAAC-3') for all promoters tested. For activated transcription we added $200 \mathrm{ng}$ of recombinant fulllength Gcn 4 that was purified as described ${ }^{40}$. HIS 4 and TMT1 template plasmids were as described ${ }^{40}$. The ACT1 template plasmid was generated by inserting the ACT1 promoter sequence ( $425 \mathrm{bp}$ upstream to $24 \mathrm{bp}$ downstream of the start codon) in pBluescript KS+ with HindIII and BamHI.

Preparation of recombinant $S p$ head module. The complete $S p$ head module was expressed in cells co-transformed with the three plasmids depicted in Supplementary Fig. 2 d. The crystallized Sp head module was expressed in cells co-transformed with the three plasmids shown in Supplementary Fig. 2e. Cells were lysed by sonication in buffer $\mathrm{E}$ (20 mM Tris- $\mathrm{HCl}, \mathrm{pH} 8.0,500 \mathrm{mM} \mathrm{NaCl}, 10 \%$ (v/v) glycerol, $5 \mathrm{mM}$ DTT) containing protease inhibitors ${ }^{12}$. After centrifugation, the supernatant was precipitated with $35 \%(\mathrm{v} / \mathrm{v})$ saturated ammonium sulphate, and pellets were resuspended in buffer $\mathrm{E}$ and loaded on a $2 \mathrm{ml} \mathrm{Ni}$-NTA agarose beads column (QIAGEN) equilibrated in buffer E. The flow-through was reloaded on another $2 \mathrm{ml} \mathrm{Ni-NTA}$ column and both columns were washed with buffer E containing increasing concentration of imidazole $(0,10$ and $20 \mathrm{mM})$. The complex was eluted from both columns with buffer E containing $300 \mathrm{mM}$ imidazole. The His-tag was cleaved overnight with $0.7 \mathrm{U}$ thrombin (Sigma-Aldrich) per mg of protein while dialysing against buffer $\mathrm{F}$ (20 mM Tris-HCl, pH 8.0, $150 \mathrm{mM} \mathrm{NaCl}, 2 \mathrm{mM}$ DTT). The complex was further purified by anion exchange chromatography using a $1 \mathrm{ml}$ HiTrap Q HP column (GE Healthcare) equilibrated in buffer G (20 mM Tris- $\mathrm{HCl}$, $\mathrm{pH} 8.0,50 \mathrm{mM} \mathrm{NaCl}, 10 \%$ (v/v) glycerol, $2 \mathrm{mM} \mathrm{DTT})$ and eluted with a linear gradient from $50 \mathrm{mM}$ to $500 \mathrm{mM} \mathrm{NaCl}$ in buffer $\mathrm{G}$ over 70 column volumes. Fractions containing the complex were collected and applied to a HiLoad 16/600 Superdex 200 pg (GE Healthcare) size exclusion column equilibrated in buffer F. The $S p$ head module was concentrated to $6 \mathrm{mg} \mathrm{ml}^{-1}$, flash-frozen and stored at $-80^{\circ} \mathrm{C}$. The identity of the polypeptides was confirmed by mass spectrometry. Crystal structure determination of $\boldsymbol{S p}$ Mediator head module. Crystals of the native complex were grown at $3.5 \mathrm{mg} \mathrm{ml}^{-1} \mathrm{Sp}$ head module at $20{ }^{\circ} \mathrm{C}$ in hanging drops over reservoirs containing $50 \mathrm{mM}$ MES, pH 6.0, and $1 \mathrm{M}$ ammonium sulphate. Crystals were collected and transferred in a stepwise manner to the final cryo-solution (50 mM MES, pH 6.0, 100\% saturated lithium sulphate) and flashfrozen in liquid nitrogen. For structure solution native crystals were derivatized with $\mathrm{Ta}_{6} \mathrm{Br}_{12}$ (refs 41-43) (Proteros biostructures) and Yb-DTPA-BMA ${ }^{44}$ (NatXray). $\mathrm{Ta}_{6} \mathrm{Br}_{12}$ was added directly to the crystallization drop at $2 \mathrm{mM}$ for $1 \mathrm{~h}$. Yb-DTPA-BMA was added to the final cryo-solution at $100 \mathrm{mM}$ for $10 \mathrm{~min}$ and back-soaked $10 \mathrm{~s}$ before freezing. The $S p$ head module was further labelled with selenomethionine as described ${ }^{33}$. Crystals of the labelled protein were grown at $2 \mathrm{mg} \mathrm{ml}^{-1} \mathrm{Sp}$ head module at $20^{\circ} \mathrm{C}$ in hanging drops over a reservoir composed of $50 \mathrm{mM}$ MES, pH 6.0, 0.95 M ammonium sulphate. Crystals were measured at $100 \mathrm{~K}$ at SLS, Villigen, Switzerland. Diffraction data were collected on a PILATUS $6 \mathrm{M}$ detector at the X06SA beamline (native crystals and $\mathrm{Ta}_{6} \mathrm{Br}_{12}$ and $\mathrm{Yb}-\mathrm{DTPA}$ BMA derivatives) or on a PILATUS $2 \mathrm{M}$ detector at the X06DA beamline (selenomethionine-labelled crystals). Data were processed with XDS and XSCALE ${ }^{34}$ The HySS submodule in Phenix ${ }^{35}$ identified four heavy-atom sites in the $\mathrm{Ta}_{6} \mathrm{Br}_{12}$ derivative and a single heavy-atom site in the $\mathrm{Yb}$-DTPA-BMA derivative. These sites were used as input for MIRAS phasing with autoSHARP ${ }^{45}$. Density modification was performed with Resolve $^{46}$ and yielded an interpretable electron density map. In parallel, an anomalous difference Fourier map was calculated with data from selenomethionine-labelled crystals and with experimental MIRAS phases. The experimental map, in combination with the selenomethionine 
sequence markers, allowed the unambiguous manual fitting of the amino-terminal part of Med6 (residues 10-117) and of the Med8C-Med18 subcomplex (PDB code 3C0T). Guided by our knowledge of the folds and topologies of the Med11NMed22N and Med17C-Med11C-Med22C subcomplexes, we could build a polyalanine model corresponding to all remaining secondary structure elements with $\mathrm{COOT}^{36}$. MIRAS phases were then combined with phases from this initial model and density was modified using the AutoBuild routine in Phenix ${ }^{35}$. This led to an improved electron density map that allowed for an extension of the initial model. This process was iterated until no further improvement of the map was observed. Then, 23 selenium sites were identified and used as input for SAD phasing with the AutoSol routine in Phenix ${ }^{35}$. The new phases were combined with phases from the partial model. After density modification, an electron density map of excellen quality was obtained, in which many side chains and most of the linkers between secondary structure elements were visible. After rebuilding, the model was refined with autoBUSTER ${ }^{47}$. The resulting $2 F_{\mathrm{o}}-F_{\mathrm{c}}$ electron density map allowed further improvement of the model. After several rounds of rebuilding and refinement with autoBUSTER and Phenix.refine ${ }^{35}$ using individual B-factor and TLS refinement, we obtained the final model, which shows good stereochemistry and an $R_{\text {free }}$ value of $25.8 \%$ (Supplementary Table 2). Including high-resolution data ${ }^{37}$ resulted in a refined structure with better $R_{\text {free }}$ values and stereochemistry. More than $95 \%$ and $99 \%$ of the residues fall in favoured and allowed regions of the Ramachandran plot, respectively ${ }^{38}$.

Modelling of the $S c$ Mediator head module. A hybrid model was build using our structure of Sc Med17C-Med11C-Med22C and the structure of Sc Med8CMed18-Med20 (ref. 11) as models for the fixed and moveable jaws, respectively. For the neck, our $S p$ head module was used, replacing Med11N and Med22 helix $\alpha 1$ with the corresponding $S c$ elements in the $S c$ Med11N-Med22N structure ${ }^{12}$ Other amino acids of the neck were replaced with their $S c$ counterparts, based on sequence alignments. Hydrophobic character of the amino acid situated in the core in the resulting neck model was systematically checked. The obtained models for the two jaws, the spine and the shoulder were separately fitted onto the corresponding elements in the published architectural model of the $S c$ head module ${ }^{10}$ ( $\mathrm{PDB}$ code $3 \mathrm{RJ} 1$ ), using secondary structure matching in $\mathrm{COOT}^{36}$. This resulted in a model that explained most of the published electron density. Regions of the model that lacked convincing density were removed, as well as side-chain atoms. The resulting model was adjusted by rigid-body refinement with Phenix.refine ${ }^{35}$, using the six structural elements present in the model (partial shoulder, partial arm, spine, tooth, nose and moveable jaw) as separate rigid-body groups. For figure preparation and structure interpretation, $S p$ Med20 was modelled with MODELLER $^{48}$.

31. Edgar, R. C. MUSCLE: multiple sequence alignment with high accuracy and high throughput. Nucleic Acids Res. 32, 1792-1797 (2004)

32. Gouet, P., Courcelle, E., Stuart, D. I. \& Metoz, F. ESPript: analysis of multiple sequence alignments in PostScript. Bioinformatics 15, 305-308 (1999).

33. Budisa, N. et al. High-level biosynthetic substitution of methionine in proteins by its analogs 2-aminohexanoic acid, selenomethionine, telluromethionine and ethionine in Escherichia coli. Eu. J. Biochem. 230, 788-796 (1995).

34. Kabsch, W. Xds. Acta Crystallogr. D 66, 125-132 (2010).

35. Adams, P. D. et al. PHENIX: a comprehensive Python-based system for macromolecular structure solution. Acta Crystallogr. D 66, 213-221 (2010).

36. Emsley, P., Lohkamp, B., Scott, W. G. \& Cowtan, K. Features and development of Coot. Acta Crystallogr. D 66, 486-501 (2010).

37. Karplus, P. A. \& Diederichs, K. Linking crystallographic model and data quality. Science 336, 1030-1033 (2012).

38. Chen, V. B. et al. MolProbity: all-atom structure validation for macromolecular crystallography. Acta Crystallogr. D 66, 12-21 (2010).

39. Sheldrick, G. M. A short history of SHELX. Acta Crystallogr. A 64, 112-122 (2008).

40. Seizl, M. et al. A conserved GA element in TATA-less RNA polymerase II promoters. PLOS ONE 6, e27595 (2011).

41. Schneider, G. \& Lindqvist, Y. Ta6Br14 is a useful cluster compound for isomorphous replacement in protein crystallography. Acta Crystallogr. D 50, 186-191 (1994)

42. Knäblein, J. et al. $\mathrm{Ta}_{6} \mathrm{Br}_{12}{ }^{2+}$, a tool for phase determination of large biological assemblies by X-ray crystallography. J. Mol. Biol. 270, 1-7 (1997).

43. Cramer, P. et al. Architecture of RNA polymerase II and implications for the transcription mechanism. Science 288, 640-649 (2000).

44. Girard, E., Stelter, M., Vicat, J. \& Kahn, R. A new class of lanthanide complexes to obtain high-phasing-power heavy-atom derivatives for macromolecular crystallography. Acta Crystallogr. D 59, 1914-1922 (2003).

45. Vonrhein, C., Blanc, E., Roversi, P. \& Bricogne, G. Automated structure solution with autoSHARP. Methods Mol. Biol. 364, 215-230 (2007).

46. Terwilliger, T. C. Maximum-likelihood density modification. Acta Crystallogr. D 56, 965-972 (2000).

47. Blanc, E. et al. Refinement of severely incomplete structures with maximum likelihood in BUSTER-TNT. Acta Crystallogr. D Biol 60, 2210-2221 (2004).

48. Eswar, N. et al. Comparative protein structure modeling using MODELLER. Curr. Protoc. Bioinformatics Chapter 5, 5.6.1 (2006). 